\title{
Efficient Non-Doped Deep Blue Organic Light Emitting Diodes with High External Quantum Efficiency and a Low Efficiency Roll-off Based on Donor- Acceptor Molecules
}

\author{
Amjad Islam $^{\ddagger}$ a,b , Qiang Wang ${ }^{\ddagger c}$, Lei Zhang ${ }^{a}$, Tao Lei ${ }^{a}$, Ling Hong ${ }^{\text {a }}$, Rongjuan Yang ${ }^{\text {a }}$, Zhiyang \\ Liu $^{\text {a }}$, Ruixiang Peng ${ }^{\text {a, }}{ }^{\text {, Liang-Sheng Liao }}{ }^{{ }^{*}}$, Ziyi Ge $^{\text {a } *}$ \\ ${ }^{a}$ Ningbo Institute of Materials Technology \& Engineering, Chinese Academy of Sciences, Ningbo 315201, P. R. \\ China \\ ${ }^{b}$ University of Chinese Academy of Sciences, Beijing-100049, P.R. China \\ ${ }^{c}$ Institute of Functional Nano \& Soft Materials (FUNSOM), Soochow University, Suzhou, Jiangsu 215123, P.R. \\ China \\ ${ }^{d}$ Jiangsu Collaborative Innovation Center of Photovoltaic Science and Engineering, Changzhou 213164, P. R. \\ China \\ ${ }^{\ddagger}$ These authors contributed equally \\ *Corresponding Author: geziyi@nimte.ac.cn (Ziyi Ge), 1sliao@suda.edu.cn (Liang-Sheng Liao)
}

\begin{abstract}
:
Two phenanthroimidazole derivatives containing carbazole and pyrene units were designed and synthesized. Highly efficient non-doped deep blue organic light emitting diodes (OLEDs) were fabricated by using these materials as emissive layer. The non-doped devices demonstrated high efficiency $(4.24 \mathrm{~cd} / \mathrm{A}, 3.67 \mathrm{~lm} / \mathrm{W}, 4.66 \%)$, low efficiency roll-off at higher current densities, and stable deep blue emissions with CIEy around 0.10, which are excellent results for deep-blue OLED devices. This study reveals that the combination of phenanthroimidazole and donorlinker-acceptor strategy has a great potential for developing high performance deep-blue OLEDs.
\end{abstract}


Keywords: Blue emission, phenanthroimidazole, pyrene, carbazole, non-doped, OLEDs

\section{Introduction}

Organic light emitting diodes (OLEDs) have attracted much attention in the past decades for their promising application potentials in flat panel display and solid-state lighting resources. Recently, blue OLEDs have gained a lot of scientific and industrial interest because of their short life time and low efficiency compared to the green and red ones [1]. Blue emission not only decreases the power consumption but it also produces emission of other colors through cascade energy transfer mechanism [2], whereas highly efficient and stable deep blue emitters are still deficient [3-4]. Several phosphorescent and thermally activated delayed fluorescence (TADF) materials with excellent performance are developed which can achieve 100\% IQE [5-7]. However, a very less number of these materials fulfills the requirement of deep blue emitters with Commission International de L'Eclairage (CIE) coordinates of $y<0.08$, which meets the National Television System Committee (NTSC) standard blue CIE of $(0.14,0.08)$ [8-11]. A difficulty in the synthesis of efficient phosphor is the non-radiative decay through the metal dorbitals during metal-ligand charge transfer (MLCT) band into deep blue region [12-13]. Due to intramolecular charge transfer (ICT), TADF materials show broad emission, leading to a decrease in color purity [14]. So, development of efficient novel deep blue emitters is still of great importance.

Inspite of high efficiency, host-guest system has some major drawbacks. For instance, the phase separation could diminish the device performance badly during operation. Apart from this, it requires the additional further design of good hosts is required which makes it difficult to evaluate the blue emitters. In view of this, use of non-doped emissive materials are a rational 
approach for OLED applications. Several attempts have been made to explore different types of non-doped blue fluorescent emitters, for instance anthracene, fluorene, spirobifluorene, di(styryl)arylene, and pyrene containing molecules to enhance their electroluminescence (EL) performances [15-18]. For example, Adachi and co-workers developed 9,10-diphenylanthracene which is also used as a standard to measure the photoluminescent quantum yield (PLQY) because its fluorescence quantum efficiency is near one in dilute solution [19]. But, its fluorescence is easily quenched due to aggregation in the solid state.

Another approach to synthesize efficient non-doped blue emitters is the introduction of bulky side chain in the emission core. By this way, fluorescence quantum yield of the material is increased in the thin film state. With this idea, different hydrocarbons with steric hindrance were attached to the anthracene core to obtain non-doped blue emitters $[16,18]$. Apart from good thermal stability and ability to reduce the close packing in film, good charge injection and charge transporting properties for balanced charge recombination are also required to develop high performance non-doped blue emitters. Different groups with good charge transport ability were incorporated into the molecules to improve its charge transport properties [20-21]. Non-doped blue emissive materials with high PLQY, restricted intermolecular interactions, and good charge injection as well as charge transporting abilities are demanded to obtain stable and efficient blue OLED devices.

Besides this, there is one more obstacle that restricts the commercialization of OLEDs is the significant efficiency roll-off at higher current densities. Both, phosphorescent and TADF materials exhibit this effect because of triplet-triplet annihilation (TTA) process [22-23]. It is also reported that many efficient fluorophores also show significant efficiency roll-off, which is due to the reduction of carrier confinement at high current densities and unbalanced charge 
carrier mobility [24-26]. Consequently, the deep blue material with a balanced ambipolar property is urgently required to reduce the efficiency roll-off.

Imidazole is an asymmetric aromatic heterocyclic compound containing two nitrogen atoms with different points of bonding and ambipolar nature [27-28]. Imidazole based materials have demonstrated excellent performances in optoelectronic applications [29-30], not only used as electron transport materials, 1,3,5-tris(N-phenylbenzimidazol-2-yl)-benzene (TPBi), but also as efficient emissive layers, (BTPE-PI, PhBPI) [31-37]. Interestingly, these materials show quite low efficiency roll-off. Apart from benzimidazole and phenanthrene imidazole based, some pyrene containing blue imidazole emitters have some potential for applications in optoelectronic field [38-39]. Carbazole is one of those few molecules which have high triplet energy level ( $\left.\mathrm{E}_{\mathrm{T}}\right)$ and sufficient HT property, whose derivatives are considered as the good candidates for blue phosphorescent host materials. Pyrene is a blue-light-emitting compound containing four fused benzene units, which is a very promising building block for OLEDs because of its features such as good chemical stability, high charge mobility, and excellent hole injection ability along with high photoluminescence efficiency and high charge carrier mobility [40-41]. Importantly, pyrene itself does not work well as blue-emitter in OLEDs because it forms excimers, resulting in a redshifted emission with a decrease in the fluorescence intensity. This property has restricted the use of pyrene as an emissive material in OLEDs. Hence, many efforts in the structural modification of pyrene containing emissive materials have been made to suppress aggregation and increase the fluorescence quantum yield for OLEDs application.

Herein, to synthesize efficient blue emissive materials, we have developed two novel phenanthroimidazole emitters containing pyrene and carbazole, N,N-diphenyl-4'-(1-(3-(9phenyl-9H-carbazol-3-yl)phenyl)-1H-phenanthro[9,10-d]imidazol-2-yl)biphenyl-4-amine 
(TPACzPI) and N,N-diphenyl-4'-(1-(3-(pyren-1-yl)phenyl)-1H-phenanthro[9,10-d]imidazol-2yl)biphenyl-4-amine (TPAPyPI). These materials exhibit high thermal stability with good charge carrier properties. Non-doped OLEDs fabricated by using TPACzPI or TPAPyPI as emitting layers showed stable deep blue emission with CIEy around 0.08, high efficiency $(4.24 \mathrm{~cd} / \mathrm{A}$, $3.67 \mathrm{~lm} / \mathrm{W}, 4.66 \%)$ and a low efficiency roll-off. These results have indicated that the combination of imidazole and donor- $\pi-$-acceptor can provide a good strategy to develop stable and highly efficient deep blue emitters for OLEDs.

\section{Experimental Section}

\subsection{General Methods}

Nuclear magnetic resonance $\left({ }^{1} \mathrm{H}\right.$ NMR $)$ and $\left({ }^{13} \mathrm{C}\right.$ NMR $)$ spectra were carried out on a Bruker DRX-400 spectrometer. ESI-Ms spectra were studied with an FINNIGAN Trace DSQ mass spectrometer at $70 \mathrm{eV}$. Elemental analyses were investigated with a Perkine Elmer $2400 \mathrm{II}$ elemental analyzer. UV/vis absorption spectra were measured on a Perkine Elmer Lambda 950 spectrophotometer. Photoluminescent (PL) spectra were measured with a FLSP920 spectrophotometer. Thermogravimetric analyses (TGA) were explored using a Perkine Elmer Pyris thermogravimeter at $10^{\circ} \mathrm{C} \mathrm{min}^{-1}$ using nitrogen atmosphere. Cyclic voltammetry (CV) measurements were recorded on a CHI 660D electrochemical workstation using a Pt disk as the working electrode, a Pt wire as the counter electrode, and $\mathrm{Ag} / \mathrm{AgCl}$ as the reference electrode, in a dichloromethane solution containing $0.1 \mathrm{M}$ of tetrabutylammonium hexafluorophosphate as the supporting electrolyte.

\subsection{Synthesis of the Compounds}


Phenanthrene-9,10-dione (1), 9-phenyl-9H-carbazol-3-ylboronic acid (4), pyren-1-ylboronic acid (5), 3-bromoaniline were purchased from TCI co. 4'-(diphenylamino)biphenyl-4-carbaldehyde (2) was prepared according to the reported method [42]. 


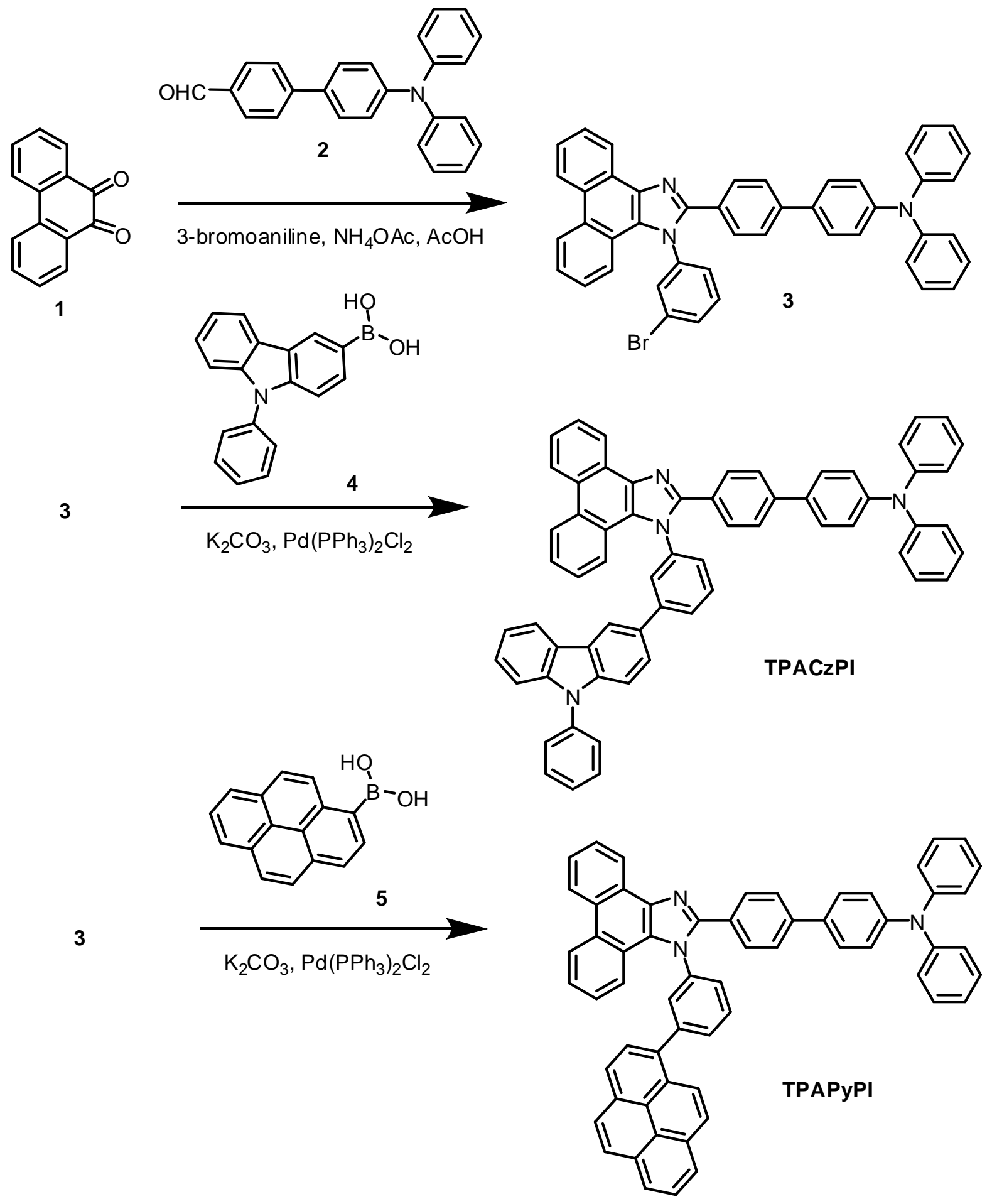

Scheme 1. The synthetic route to TPA-CzPI, TPA-PyPI.

2.2.1. Synthesis of 4'-(1-(3-bromophenyl)-1H-phenanthro[9,10-d]imidazol-2-yl)-N,N-

diphenylbiphenyl-4-amine (3): 
4'-(diphenylamino)biphenyl-4-carbaldehyde $(1.0 \mathrm{mmol}), 0.208 \mathrm{~g}$ of phenanthrene-9,10-dione (1.0 mmol), $0.40 \mathrm{~g}$ of ammonium acetate $(5.0 \mathrm{mmol}), 0.85 \mathrm{~g}$ of 3 -bromoaniline $(5.0 \mathrm{mmol})$, and acetic acid $(10 \mathrm{~mL})$ were put into a flask and refluxed under nitrogen. After about $12 \mathrm{~h}$, the reaction was stopped and cooled, and the mixture was added $50 \mathrm{~mL}$ of water and filtered. The precipitates were dissolved in dichloromethane and dried over anhydrous $\mathrm{MgSO}_{4}$. The product was purified by column chromatography. ${ }^{1} \mathrm{H}$ NMR $\left(400 \mathrm{MHz}, \mathrm{CDCl}_{3}\right): \delta 8.90(\mathrm{~d}, J=8,1 \mathrm{H})$, $8.79(\mathrm{~d}, J=8,1 \mathrm{H}), 8.72(\mathrm{~d}, J=8,1 \mathrm{H}), 7.75-7.82(\mathrm{~m}, 3 \mathrm{H}), 7.63-7.71(\mathrm{~m}, 3 \mathrm{H}), 7.49-7.57(\mathrm{~m}, 7 \mathrm{H})$ 7.27-7.36 (m, 5H), $7.20(\mathrm{~d}, J=8,1 \mathrm{H}), 7.14(\mathrm{~d}, J=8,6 \mathrm{H}), 7.04(\mathrm{t}, J=8,2 \mathrm{H})$.

\subsubsection{Synthesis of Compound TPA-CzPI:}

Compound 3 (0.5 mmol), 9-phenyl-9H-carbazol-3-ylboronic acid (4) $(1 \mathrm{mmol})$, and $50 \mathrm{mg}$ of $\operatorname{Pd}\left(\mathrm{PPh}_{3}\right)_{2} \mathrm{Cl}_{2}(0.1 \mathrm{mmol})$ were added in the solution of $30 \mathrm{~mL}$ of THF and $10 \mathrm{~mL}$ of $2 \mathrm{M} \mathrm{K}_{2} \mathrm{CO}_{3}$, and the mixture was heated to $80{ }^{\circ} \mathrm{C}$ and stirred for overnight under nitrogen. After cooling, the mixture was extracted by using dichloromethane $(40 \mathrm{~mL} \times 3$ times), washed by using water $(10$ $\mathrm{mL} \times 3$ times), and dried with anhydrous $\mathrm{MgSO}_{4}$. The product was purified by silica gel column chromatography with dichloromethane-petroleum ether as an eluent, a white solid was obtained. Yield: 70\%. ${ }^{1} \mathrm{H}$ NMR (400 MHz, CDCl3): $\delta 8.95(\mathrm{~d}, \mathrm{~J}=8,1 \mathrm{H}), 8.83(\mathrm{~d}, \mathrm{~J}=8,1 \mathrm{H}), 8.74(\mathrm{~d}, \mathrm{~J}=$ 8, 1H), $8.35(\mathrm{~s}, 1 \mathrm{H}), 8.12(\mathrm{~d}, \mathrm{~J}=8,1 \mathrm{H}), 8.01(\mathrm{~d}, \mathrm{~J}=8,1 \mathrm{H}), 7.93(\mathrm{~s}, 1 \mathrm{H}), 7.42-7.79(\mathrm{~m}, 22 \mathrm{H})$, 7.25-7.36 (m, 6H), 7.10-7.14 (m, 6H), 7.03-7.06 (t, 2H). ${ }^{13} \mathrm{C}$ NMR $\left(400 \mathrm{MHz}, \mathrm{CDCl}_{3}\right) \delta$ 150.71, $147.57,144.10,141.46,140.89,140.79,139.19,137.42,133.92,131.38,130.51,129.98,129.87$ $129.43,129.35,129.29,128.43,128.36,128.12,127.70,127.66,127.56,127.05,126.83,126.44$, $126.40,126.31,125.70,125.22,124.98,124.50,124.17,124.04,123.73,123.21,123.09,123.04$ 
122.96, 121.08, 120.45, 120.28, 118.94, 110.29, 110.01. HRMS (MALDITOF), m/z: 855.30 $\left(\mathrm{M}^{+}\right)$

\subsubsection{Synthesis of Compound TPA-PyPI:}

Compound 3 (0.5 mmol), 1-pyreneboronic acid (5) $(1 \mathrm{mmol})$, and $50 \mathrm{mg}$ of $\mathrm{Pd}\left(\mathrm{PPh}_{3}\right)_{2} \mathrm{Cl}_{2}(0.1$ mmol) were added in the solution of $30 \mathrm{~mL}$ of THF and $10 \mathrm{~mL}$ of $2 \mathrm{M} \mathrm{K}_{2} \mathrm{CO}_{3}$, and the mixture was heated to $80{ }^{\circ} \mathrm{C}$ and stirred for overnight under nitrogen. After cooling, the mixture was extracted by using dichloromethane ( $40 \mathrm{~mL} \times 3$ times), washed by using water $(10 \mathrm{~mL} \times 3$ times), and dried with anhydrous $\mathrm{MgSO}_{4}$. The product was purified by silica gel column chromatography with dichloromethane-petroleum ether as an eluent, a white solid was obtained. Yield: $70 \% .{ }^{1} \mathrm{H}$ NMR (400 MHz, CDCl3): $\delta 8.96(\mathrm{~s}, 1 \mathrm{H}), 8.83(\mathrm{~d}, J=8,1 \mathrm{H}), 8.74(\mathrm{~d}, J=8,1 \mathrm{H})$, 8.19-8.22 (m, 2H), 8.07-8.12 (m, 3H), 7.98-8.02 (m, 2H), 7.75-7.92 (m, 10H), $7.67(\mathrm{~d}, J=8$, 3H), 7.57-7.62 (m, 4H), $7.44(\mathrm{t}, J=8,1 \mathrm{H}), 7.27-7.32(\mathrm{~m}, 6 \mathrm{H}), 7.17-7.20(\mathrm{~m}, 6 \mathrm{H}), 7.05(\mathrm{t}, J=8$, 2H). ${ }^{13} \mathrm{C}$ NMR (400 MHz, $\left.\mathrm{CDCl}_{3}\right) \delta 150.88,147.71,147.59,143.58,141.06,140.98,138.63$, 135.44, 133.93, 131.82, 131.38, 131.12, 131.08, 130.75, 130.23, 130.15, 129.46, 129.41, 129.34, $128.44,128.33,128.09,128.02,127.92,127.78,127.32,127.30,126.43,126.36,126.14,125.71$, $125.41,125.05,124.86,124.75,124.69,124.63,124.56,124.22,123.79,123.68,123.39,123.12$ 123.03, 122.94, 121.05; HRMS (MALDITOF), m/z: $814.20\left(\mathrm{M}^{+}\right)$.

\subsection{Device Fabrication and Characterization}

Before the fabrication of OLEDs, sublimation of the compounds TPA-CzPI and TPA-PyPI were performed with the first pipe heating up to $360^{\circ} \mathrm{C}$ for purification. The ITO-coated glass substrates were washed as usual with different solvents and then treated with UV-ozone for 15 min. The OLED devices were constructed with the structure of ITO/HAT-CN(5 nm)/NPB (40 
nm)/TAPC (10 nm)/TPA-CzPI or TPA-PyPI (30 nm)/TPBi (40 nm)/LiF (1 nm)/Al (150 nm)

(devices A and B). All the organic and inorganic layers were deposited in sequence at $10^{-6}$ Torr with the deposition rate of $0.9-1.1 \AA \mathrm{s}^{-1}$. The electroluminescence spectra and the Commission Internationale del'Eclairage coordination of the device were tested on a PR655 spectra scan spectrometer. The luminance-current and density-voltage characteristics were measured simultaneously from the measurement of the EL spectra by combining the spectrometer with a Keithley 2400 programmable voltage-current source. All measurements were conducted at room ambient conditions.

\section{Results and Discussions}

\subsection{Synthesis}

Scheme 1 illustrated the synthesis of TPA-CzPI and TPA-PyPI. Our design of the phenanthroimidazole based compounds was inspired by the twisted structure of phenanthroimidazole derivatives, widely used materials for fluorescent OLEDs that exhibit good blue emission [42]. To enhance the family of twisted phenanthroimidazole-based molecules with high performance of OLEDs, we adopted phenanthroimidazole and its derivatives as the central core to form twisted structure. Apart from this, recently we have also reported similar type of materials with extension of conjugation through hybrid local and charge transfer mechanism [37]. So, based on our previous work, two novel twisted phenanthroimidazole derivatives were designed with the same building blocks but different side chain moieties. As shown in Scheme 1, for the synthesis of these materials, an important intermediate compound (3) was prepared by the condensation of phenanthrene-9,10-dione, 4'-(diphenylamino)biphenyl-4-carbaldehyde and 3bromoaniline. Both compounds, TPACzPI and TPAPyPI were easily synthesized in high yield (more than 70\%) through suzuki coupling reaction of compound $\mathbf{3}$ with 9-phenyl-9H-carbazol-3- 
ylboronic acid (4) or pyrene-boronic acid (5), respectively. All the compounds were characterized by ${ }^{1} \mathrm{H}$ and ${ }^{13} \mathrm{C}$ NMR spectrometry as well as mass spectrometry.

\subsection{Electrochemical Properties}

The electrochemical behavior of both the materials was studied by cyclic voltammetry (CV). The HOMO energy levels of the materials were determined from the onset potential of the first oxidation relative to ferrocene (Fig. 1). The first oxidation event was observed at 0.457 and $0.436 \mathrm{eV}$ (vs.Fc/Fc+). $\mathrm{HOMO}$ values are obtained from the formula of $\mathrm{E}_{\mathrm{HOMO}}=-\left(\left[\mathrm{E}_{\mathrm{onset}}\right]_{\mathrm{ox}}+\right.$ 4.8). The values of TPACzPI and TPAPyPI are -5.01 and $-4.99 \mathrm{eV}$, respectively. These HOMO energy levels are comparatively lower than the work function of ITO, which can significantly decrease the hole injection barrier into the emission layer and enhance the device efficiency [44]. The energy band gaps ( $\mathrm{E}_{\mathrm{g}}$ )of TPACzPI and TPAPyPI were calculated to be about $3.03 \mathrm{eV}$ for each material, which are determined from the onset of UV-vis absorption spectra. Finally, the LUMO levels of TPACzPI and TPAPyPI were found to be -1.99 and $-1.96 \mathrm{eV}$. The results are summarized in Table 1. 


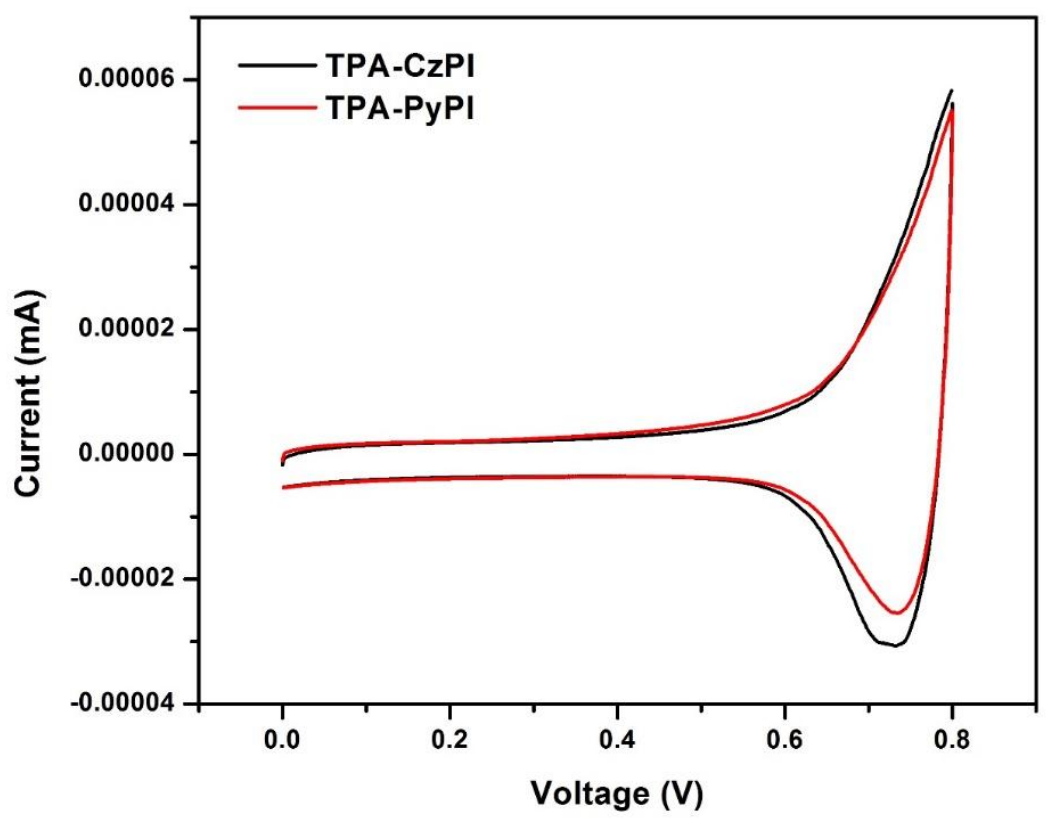

Fig. 1. Cyclic voltammogram curve of TPA-CzPI and TPA-PyPI.

\subsection{Thermal Properties}

The thermal properties of TPACzPI and TPAPyPI were investigated with TGA under a nitrogen atmosphere as shown in Fig. 2, and their thermal data are summarized in Table 1. The TGA measurement shows that both derivatives possess high thermal stabilities of more than $500^{\circ} \mathrm{C}$. A weight loss of $5 \%\left(\mathrm{~T}_{\mathrm{d}}\right)$ is observed at $528^{\circ} \mathrm{C}$ for each material. This value is $100^{\circ} \mathrm{C}$ higher than the sublimation temperature and indicates the high thermal stability of these materials. 


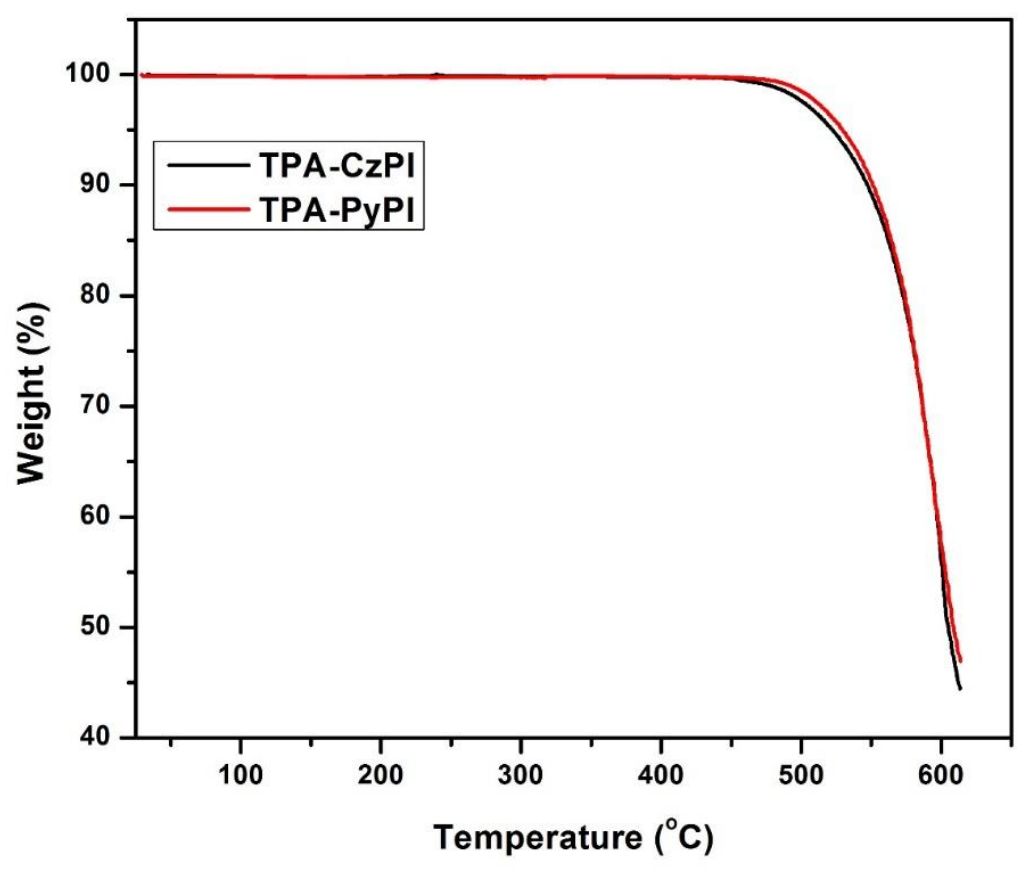

Fig. 2. TGA curves of TPA-CzPI and TPA-PyPI

\subsection{Theoretical Calculations}

To gain insight into the electronic structures of the compound, the ground-state geometries and the frontier molecular orbital energy levels were calculated using the density functional theory (DFT) in Gaussian 03 program at the B3LYP/6-31G (d) level. Both molecules are the derivatives of TPA-PPI [42], which has a twisted geometrical configuration. So, in this twisted geometry, there is a chance of overlapping of molecular orbitals to form a hybrid local and charge transfer state. From Fig. 3 (a), the calculated highest occupied molecular orbital (HOMO) of TPA-CzPI is found to be mainly distributed on the TPA moiety, phenyl linker and partly on the phenanthroimidazole moiety. Whereas the lowest unoccupied molecular orbital (LUMO) of TPACzPI is found to be mainly populated on the phenanthroimidazole moiety, phenyl linker and to some extent on TPA moiety as well (Fig. 3b). This result suggests that carbazole moiety does 
not contribute much to HOMO/LUMO levels in this molecule. On the other hand, the HOMO level of TPAPyPI is found to be mainly distributed on the TPA moiety, phenyl linker and partly on the phenanthroimidazole moiety (Fig. 3c). However, the LUMO level of TPAPyPI is localized on the pyrene moiety (Fig. 3d). The HOMO and LUMO levels of TPAPyPI are well separated. The HOMO and LUMO energy levels of TPACzPI are calculated to be -4.85 and $1.18 \mathrm{eV}$, respectively, whereas The HOMO and LUMO energy levels of TPAPyPI are calculated to be -4.92 and $-1.72 \mathrm{eV}$, respectively. Low LUMO levels of both the materials are expected to be responsible for low efficiency of the devices.

(a)

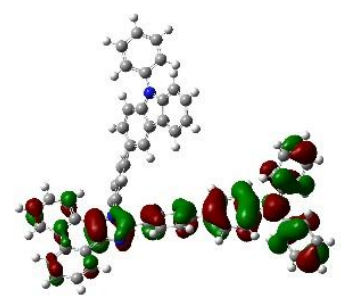

(c)

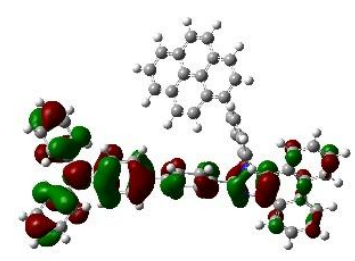

(b)

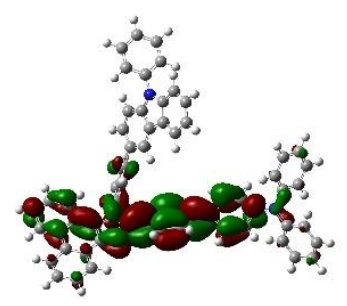

(d)

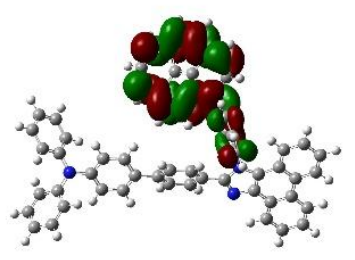

Fig. 3. Structures and HOMO LUMO levels of TPA-CzPI (a \& b) and TPA-PyPI (c \& d). 


\subsection{Photophysical Properties}

TPACzPI and TPAPyPI exhibited good solubility in common organic solvents like tetrahydrofuran (THF), dichloromethane, chloroform, etc., but were not soluble in water and methanol due to the presence of hydrophobic aromatic units. The UV-vis absorption and PL spectra of TPACzPI and TPAPyPI in solution are shown in Fig. 4, and the photophysical parameters are summarized in Table 1. The absorption spectra of both materials show two peaks at $296 \mathrm{~nm}, 360 \mathrm{~nm}, 286 \mathrm{~nm}$ and 346nm, respectively. The absorption bands in the region from 250 to $300 \mathrm{~nm}$ might originate from a combination of $\pi-\pi^{*}$ transitions of the aromatic segments [45]. The absorption peaks at $360 \mathrm{~nm}$ and $346 \mathrm{~nm}$ can be attributed to the delocalized $\pi-\pi^{*}$ transition of the phenanthroimidazole [45]. The emission spectra of TPACzPI and TPAPyPI is quite similar and gives blue emission at $441 \mathrm{~nm}$ and $442 \mathrm{~nm}$ in the solution, individually, which is very similar to their parent deep blue emitter TPAPPI (438 nm) [42]. From the onset of absorptions, the optical band gaps $\left(\mathrm{E}_{\mathrm{g}}\right)$ of TPACzPI and TPAPyPI were estimated to be $3.03 \mathrm{eV}$ of each, subsequently. 


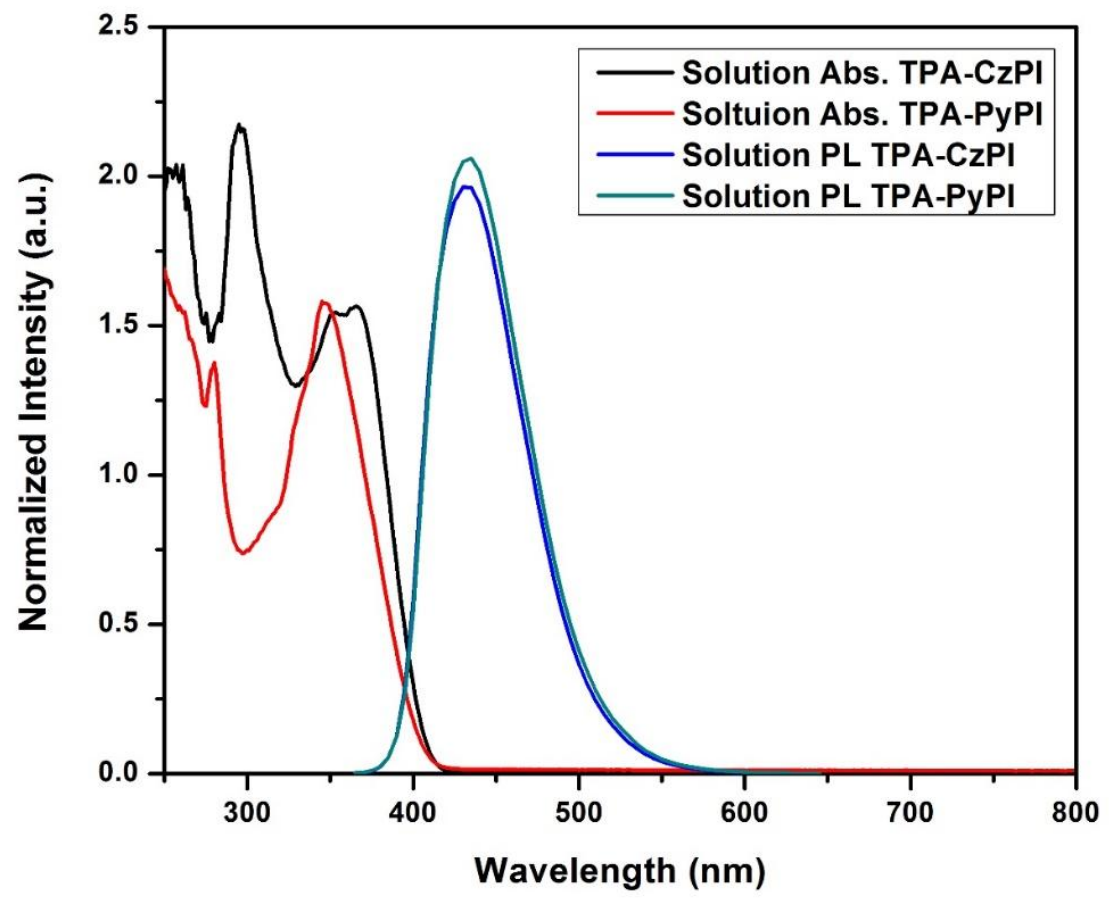

Fig. 4. UV-vis absorption and PL spectra of TPA-CzPI and TPA-PyPI in dilute THF solution.

Table: 1. Thermal properties, optional properties and energy levels of TPACzPI and TPAPyPI.

\begin{tabular}{lccccccc}
\hline Compound & Abs. $^{\mathrm{a}}(\mathrm{nm})$ & $\mathrm{PL}^{\mathrm{a}}(\mathrm{nm})$ & $\mathrm{T}_{\mathrm{d}}^{\mathrm{b}}\left({ }^{\circ} \mathrm{C}\right)$ & $\begin{array}{c}\mathrm{HOMO}\left(\mathrm{eV}^{\mathrm{c}},\right. \\
\mathrm{eV})\end{array}$ & $\begin{array}{c}\mathrm{LUMO}^{\mathrm{d}}\left(\mathrm{eV}^{\mathrm{c}},\right. \\
\mathrm{eV})\end{array}$ & $\mathrm{E}_{\mathrm{g}}^{\mathrm{e}}(\mathrm{eV})$ & $\mathrm{E}_{\mathrm{g}}^{\dagger}(\mathrm{eV})$ \\
\hline TPACzPI & 296,360 & 441 & 521 & $-5.01 /-4.85$ & $-1.99 /-1.18$ & 3.03 & 3.67 \\
TPAPyPI & 286,346 & 442 & 529 & $-4.99 /-4.92$ & $-1.96 /-1.72$ & 3.03 & 3.20 \\
\hline
\end{tabular}

${ }^{\text {a }}$ Measured in THF solution.

${ }^{b}$ Estimated based on absorption onset and cyclic-voltammetry.

${ }^{\mathrm{c}}$ DFT calculation with B3LYP/6-31G. 


\subsection{Electroluminescence Properties}

EL properties of TPACzPI and TPAPyPI were finally investigated to prove its applicable feasibility. Non-doped devices were fabricated using a device structure of ITO/HAT-CN (5 $\mathrm{nm}) / \mathrm{NPB}(40 \mathrm{~nm}) / \mathrm{TCTA}$ or TAPC $(10 \mathrm{~nm}) / \mathrm{TPACzPI}$ or TPAPyPI $(30 \mathrm{~nm}) / \mathrm{TPBi}(40 \mathrm{~nm}) / \mathrm{LiF}(1$ $\mathrm{nm}) / \mathrm{Al}(150 \mathrm{~nm})$ (devices A and B), where 1,4,5,8,9,11-hexaazatriphenylene hexacarbonitrile (HAT-CN) and LiF were used to improve hole and electron injection of ITO (indium tin oxide) anode and $\mathrm{Al}$ cathode interface, respectively, N,N'-di-1-naphthyl-N,N'-diphenylbenzidine (NPB) and 4,4',4"-tri(N-carbazolyl)-triphenylamine $\quad$ (TCTA) or $\quad 1,1^{\prime}$-bis(di-4-tolylaminophenyl)cyclohexane (TAPC) were utilized as the hole transporting layers (HTL), while 1,3,5-tris(N-phenylbenzimidazole-2-yl)benzene (TPBi) employed as the electron transporting (ETL) and hole-blocking layer (HBL).

Fig. 5 presents the current density-voltage-luminance (J-V-L), at the same current densities, TPAPyPI shows stronger fluorescence, resulting in higher efficiency compared to TPACzPI. The two fluorescent emitters also show great balance between high efficiency and high luminance, i.e., the efficiency roll-off of CE and EQE are very low as shown in Fig.6 \& 8. This small efficiency roll off is ascribed to the ambipolar nature of these derivatives. The performance data of all the devices are listed in Table 2. For non-doped devices, A1 and B1 using TCTA as EBLs, TPACzPI and TPAPyPI exhibited unaltered emission peaks at $434 \mathrm{~nm}$ and $440 \mathrm{~nm}$ and the full width at half-maximum (FWHM) values are located at 55.3 and $55.8 \mathrm{~nm}$, respectively (as shown in Fig. 4), with CIE coordinates of $(0.153,0.087)$ and $(0.151,0.082)$, which are very close to the European Broadcast Union (EBU) television blue emission (EBU, 0.150, 0.060). Moreover, the 
devices reach maximum EQEs of $3.62 \%$ and $3.99 \%$ with TPACzPI and TPAPyPI as emitter, respectively, which are among the excellent efficiencies of deep blue devices [46-49].

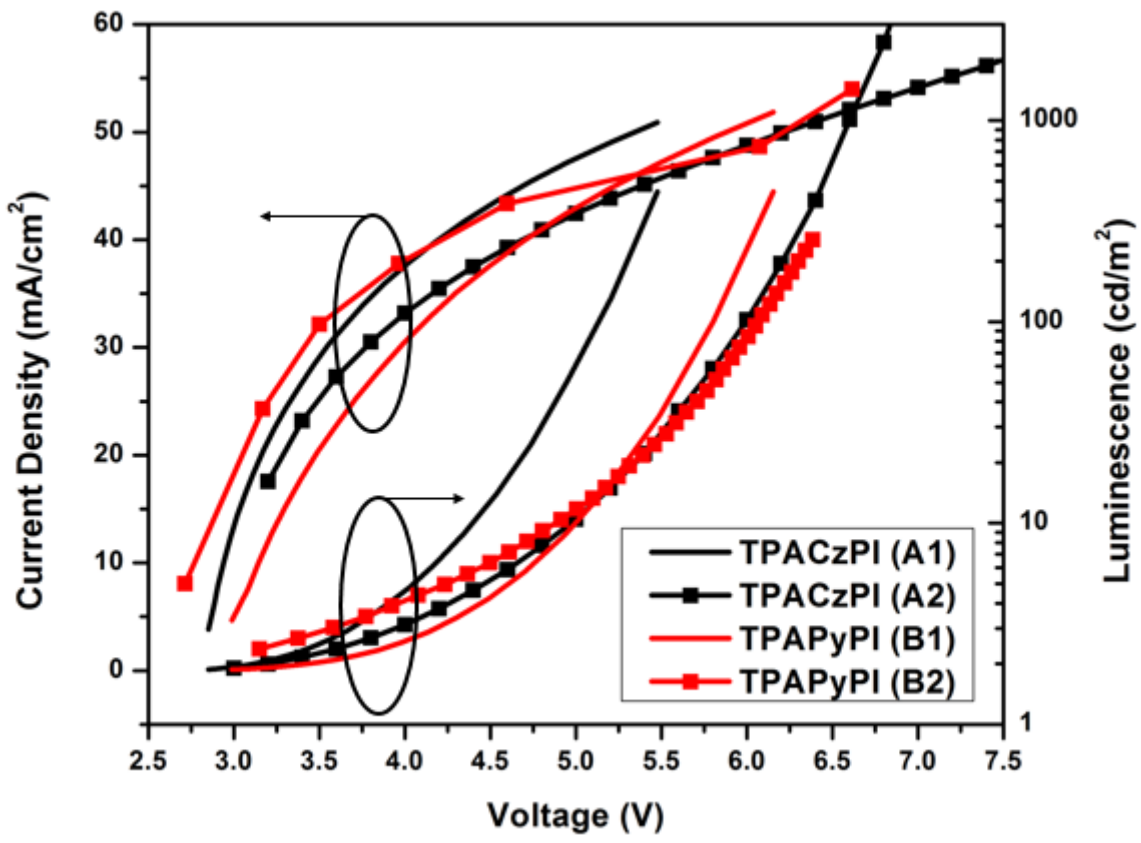

Fig. 5. Current density-voltage-luminance (J-V-L) characteristics of non-doped devices A and B.
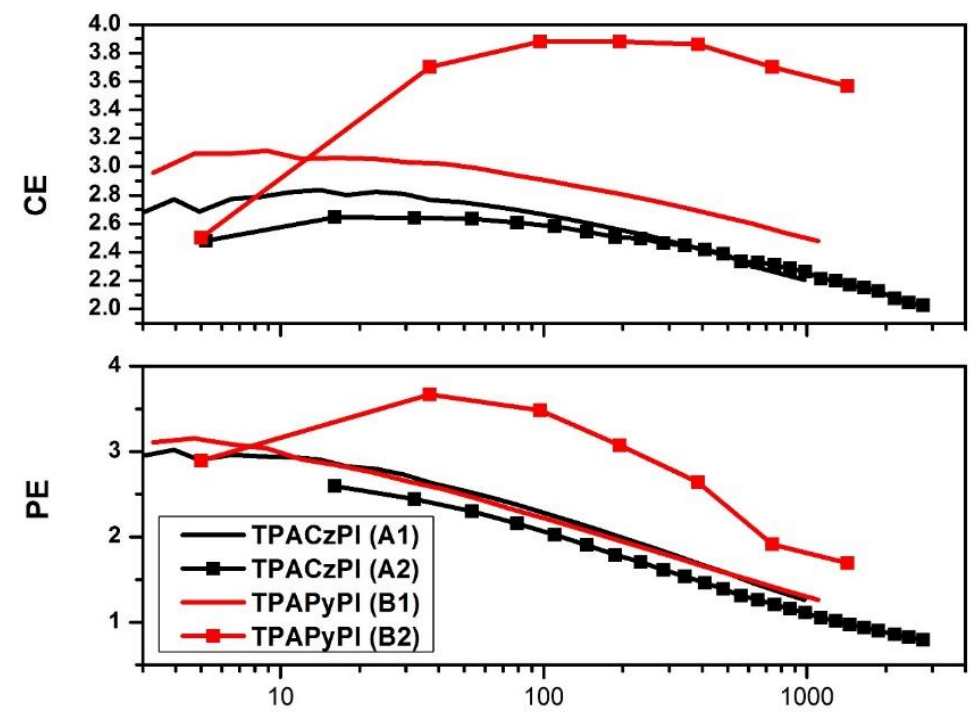

Fig. 6. Current efficiencies versus luminance and EQEs versus luminance characteristics of devices. 
To achieve better device performance, TAPC (device A2 \& B2) was used as EBL instead of TCTA (device A1 \& B1) to fabricate non-doped device with TPAPyPI. The low-lying LUMO of TCTA is likely to cause electrons diffusing from the emissive layer, which is the primary cause of low efficiencies. The high-lying LUMO and higher triplet energy $\left(\mathrm{T}_{1}=2.98 \mathrm{eV}\right)$ of TAPC compared to TCTA can confine more excitons and blocking electrons from diffusing into the HTL. The high-lying HOMO of TAPC (5.4 eV) compared with TCTA $(5.7 \mathrm{eV})$ will decrease the injection barrier between NPB/EBL interface and achieve a better energy level alignment. As the J-V characteristics (Fig. 5) shows, the device using TAPC as EBL can get higher current densities under lower driving voltage. However, the steeper increment under higher driving voltage indicates more charge carriers are injecting into the adjacent HTLs. Overall, TAPC can maintain high current density at low driving voltage, while blocking electrons diffusing into NPB. Positively, the EL (Fig. 7) of TPAPyPI device B2 remain nearly identical to TPAPyPI device B1 and exhibited excellent deep blue light with a CIE coordinate of $(0.1454,0.1367)$. As expected, the EQE increased from $3.99 \%$ to $4.66 \%$, and the PE was also increased. Furthermore, device B2 maintain high EQE of $4.39 \%$ even at a bright luminescence of $1000 \mathrm{~cd} \mathrm{~m}^{-2}$. On the other hand, the EL (Fig. 7) of TPACzPI device A2 remain nearly identical to TPACzPI device A1 also and exhibited excellent deep blue emission with a CIE coordinate of $(0.159,0.095)$. However, surprisingly, the EQE of TPACzPI device A2 is decreased from $3.62 \%$ to $3.11 \%$ (device A1). But overall, the performance of TPAPyPI based devices is much better than TPACzPI devices. This is attributed to the high photoluminescence quantum yield of pyrene based derivatives, leading to the high efficiencies of the devices. A low efficiency roll-off is found in the devices because of comparatively balanced charge carrier mobility at higher current 
density and better exciton confinement in the recombination zone (emissive layer). The nondoped device performances of these materials are better than many similar molecules such as; PPI, TPA-PIM, TPA-PPI, PyINA [46-49]. Comparison of these results with the reported ones clearly demonstrate the significant improvement not only in $\mathrm{PE}_{\max }$ but in $\mathrm{EQE}_{\max }$ as well. From the device performance of these emitters, we can expect that by the extension of chain length at the phenyl ring of TPA-PPI with fused ring units, efficient derivatives of TPA-PPI could be obtained with superior device efficiency.

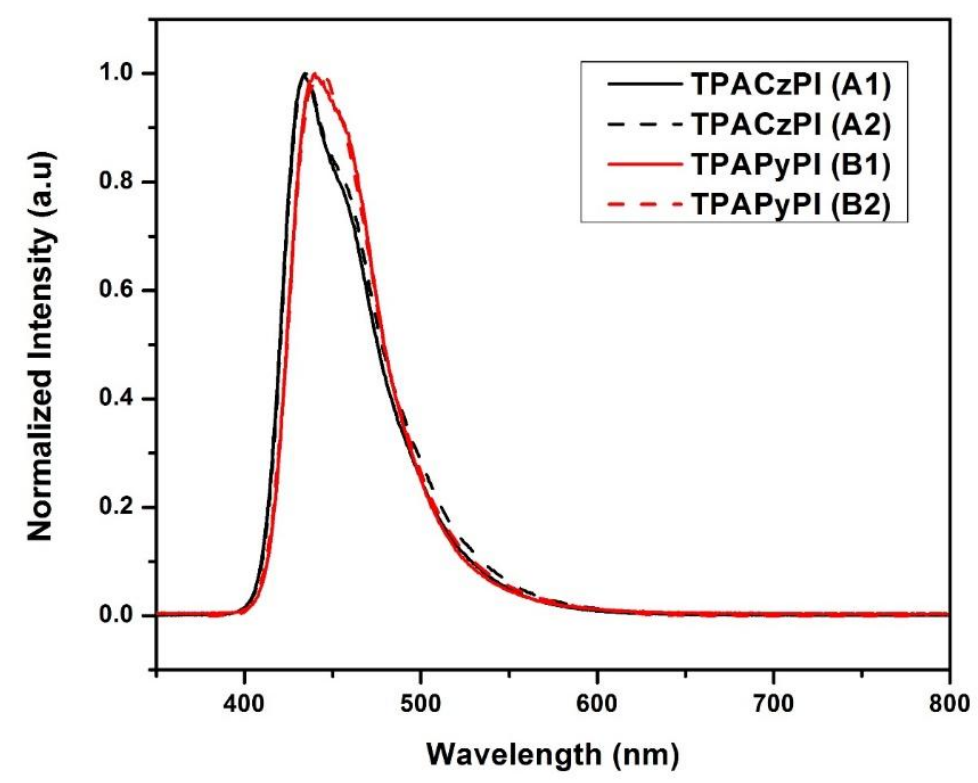

Fig. 7. EL spectra at a current density of $5 \mathrm{~mA} / \mathrm{cm}^{2}$. 


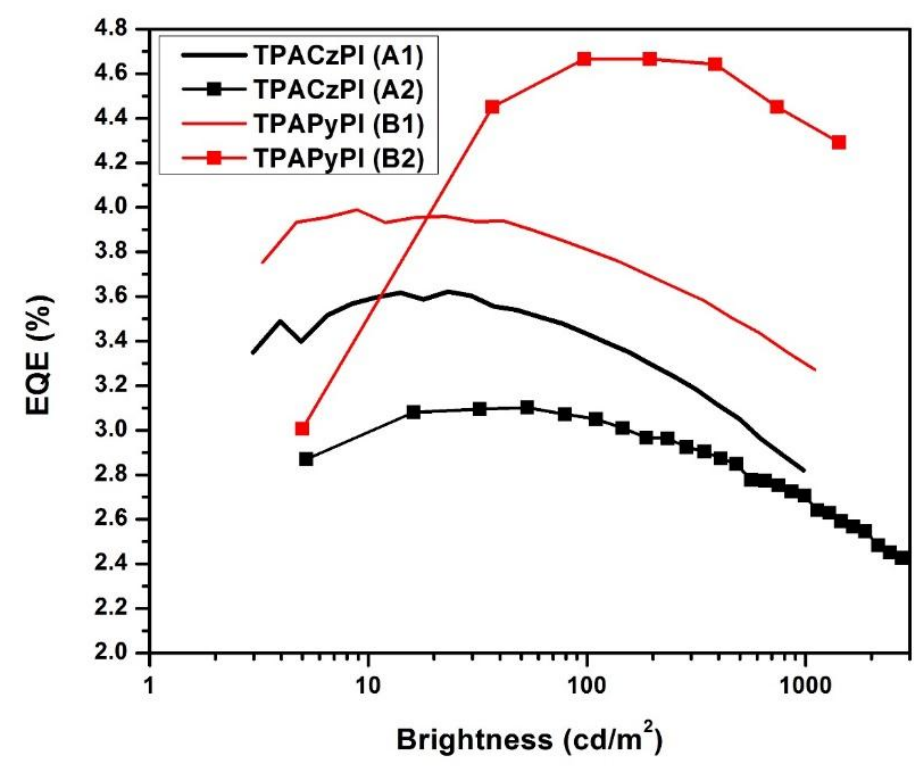

Fig. 8. Power efficiency versus current density characteristics of devices.

Table 2: Electroluminescence Data of the devices.

\begin{tabular}{|c|c|c|c|c|c|c|c|}
\hline Compound & $V_{o n}{ }^{a}(V)$ & $\begin{array}{l}C E_{\max }{ }^{b} \\
(\mathrm{~cd} / \mathrm{A})\end{array}$ & $\begin{array}{l}\mathrm{PE}_{\max }{ }^{\mathrm{c}} \\
(\mathrm{Im} / \mathrm{W})\end{array}$ & $\begin{array}{c}\mathrm{EQE}_{\max }{ }^{\mathrm{d}} \\
(\%)\end{array}$ & $\begin{array}{c}\mathrm{EL} \\
\lambda_{\max }{ }^{e}(\mathrm{~nm})\end{array}$ & $\mathrm{CIE}^{\dagger}(\mathrm{x}, \mathrm{y})$ & $\begin{array}{c}\text { EQE at } \\
100 / 1000 \\
\mathrm{~cd} / \mathrm{m}^{2}\end{array}$ \\
\hline $\mathrm{PP}^{46}$ & 3.8 & 0.71 & 0.40 & 1.86 & 412 & $\begin{array}{l}(0.161, \\
0.065)\end{array}$ & - \\
\hline TPA-PIM ${ }^{47}$ & - & 1.14 & 0.79 & 3.28 & 420 & $\begin{array}{l}(0.161 \\
0.046)\end{array}$ & $316 / 3.11$ \\
\hline TPA-PPI ${ }^{48}$ & - & 5.66 & 6.13 & 5.02 & 434 & $\begin{array}{l}(0.15 \\
0.11)\end{array}$ & 3.76 \\
\hline PyINA ${ }^{49}$ & 2.9 & 2.62 & - & 3.68 & 440 & (0.157, & $3.61 / 3.66$ \\
\hline
\end{tabular}




\begin{tabular}{|c|c|c|c|c|c|c|c|}
\hline & & & & & & $0.084)$ & \\
\hline $\mathrm{A} 1$ & 3.67 & 2.84 & 3.02 & 3.62 & $434 / 55.3$ & $\begin{array}{l}(0.15 \\
0.08)\end{array}$ & $3.43 / 2.81$ \\
\hline $\mathrm{A} 2$ & 3.20 & 2.64 & 2.61 & 3.11 & $434 / 55$ & $\begin{array}{l}(0.15 \\
0.09)\end{array}$ & $3.05 / 2.70$ \\
\hline B1 & 4.12 & 3.12 & 3.15 & 3.99 & $440 / 55.8$ & $\begin{array}{l}(0.15 \\
0.08)\end{array}$ & $3.81 / 3.30$ \\
\hline B2 & 3.36 & 4.24 & 3.67 & 4.66 & $440 / 55$ & $\begin{array}{l}(0.14 \\
0.13)\end{array}$ & $4.64 / 4.39$ \\
\hline
\end{tabular}

\footnotetext{
${ }^{a}$ Driving voltage measured at $100 \mathrm{~cd} \mathrm{~m}^{-2} .{ }^{b}$ Maximum current efficiency. ${ }^{c}$ Maximum power efficiency. ${ }^{d}$ Maximum external quantum efficiency. ${ }^{e}$ EL peaks. ${ }^{f}$ Commission International de I'Eclairage coordinates measured at a driving voltage of 5 V. ${ }^{46-49}$ References.
}

\section{Conclusions}

In summary, we have designed and prepared high performance deep-blue phenanthroimidazole derivatives. Different moieties, such as carbazole and pyrene were attached to phenyl group of phenanthroimidazole to increase the triplet energy level and photoluminescence quantum yield. The obtained two deep-blue emitters were utilized as emissive layers in OLEDs. The devices based on these emitters exhibited low turn-on voltages (as low as $3.67 \mathrm{~V}$ ), high efficiency $(4.24 \mathrm{~cd} / \mathrm{A}, 3.67 \mathrm{~lm} / \mathrm{W}, 4.66 \%$ ), comparatively small efficiency roll-off at higher current densities, and stable deep blue emissions with CIEy $<0.10$. These are among the excellent results reported so far for deep-blue OLED devices using phenanthroimidazole and pyrene based materials. The results demonstrate a promising 
combination of phenanthroimidazole and the donor- $\pi$--acceptor to develop efficient deep blue emitters.

\section{Acknowledgment}

This work was financially supported by the National Natural Science Foundation of China (21574144 and 21674123), National Key Research and Development Plan (2016YFB0401000), Zhejiang Provincial Natural Science Foundation of China (LR16B040002), Ningbo Municipal Science and Technology Innovative Research Team (2015B11002 and 2016B10005), CAS Interdisciplinary Innovation Team, CAS Key Project of Frontier Science Research (QYZDBSSW-SYS030) and CAS Key Project of International Cooperation (174433KYSB20160065). Amjad Islam acknowledges the University of Chinese Academy of Sciences (UCAS) and The World Academy of Sciences (TWAS) for awarding the CAS-TWAS president's PhD fellowship.

\section{References}

[1] Zhu MR, Yang CL. Blue fluorescent emitters: design tactics and applications in organic lightemitting diodes. Chem Soc Rev 2013;42:4963-76.

[2] Chou HH, Chen YH, Hsu HP, Chang WH, Cheng CH. Synthesis of diimidazolystilbenes as n-type blue fluorophores: alternative dopant materials for highly efficient electroluminescent devices. Adv Mater 2012;24:5867-71

[3] C-H. Chien, C-K. Chen, F-M. Hsu, C-F. Shu, P-T. Chou, C-H. Lai. Multifunctional Deep-Blue Emitter Comprising an Anthracene Core and Terminal Triphenylphosphine Oxide Groups Adv. Funct. Mater 2009;19:560-566. 
[4] H. Kaji, H. Suzuki, T. Fukushima, K. Shizu, K. Suzuki, S. Kubo, T. Komino, H. Oiwa, F. Suzuki, A. Wakamiya, Y. Murata. C. Adachi. Purely organic electroluminescent material realizing 100\% conversion from electricity to light. Nat Commun 2015;6: 8476.

[5] H. Yersin. Highly Efficient OLEDs with Phosphorescent Materials; John Wiley \& Sons: New York, 2008.

[6] H. Uoyama, K. Goushi, K. Shizu, H. Nomura, C. Adachi. Highly efficient organic lightemitting diodes from delayed fluorescence. Nature 2012;492:234-238.

[7] L.S. Cui, Y.M. Xie, Y.K. Wang, C. Zhong, Y.L. Deng, X.Y. Liu, Z.Q. Jiang, L. S. Liao. Pure Hydrocarbon Hosts for $\approx 100 \%$ Exciton Harvesting in Both Phosphorescent and Fluorescent Light-Emitting Devices. Adv. Mater 2015;27:4213-4217.

[8] Q. Zhang, J. Shizu, K. Li, S. Huang, S. Hirata, H. Miyazaki, C. Adachi. Design of efficient thermally activated delayed fluorescence materials for pure blue organic light emitting diodes J. Am. Chem. Soc 2012;134:14706-14709.

[9] H. Liang, X. Wang, X. Zhang, Z. Ge, X. Ouyang, S. Wang. Efficient tuning of electroluminescence from sky-blue to deep-blue by changing the constitution of spirobenzofluorene derivatives. Dyes \& Pigment 2014;108:57-63.

[10] Q. Zhang, B. Li, S. Huang, H. Nomura, H. Tanaka, C. Adachi. Efficient blue organic lightemitting diodes employing thermally activated delayed fluorescence. Nat Photonics 2014;8:326-332. 
[11] J. Lee, H. F. Chen, T. Batagoda, C. Coburn, P.I. Djurovich, M.E. Thompson, S.R. Forrest. Deep blue phosphorescent organic light-emitting diodes with very high brightness and efficiency. Nat. Mater 2016;15:92-98.

[12] P-T. Chou, Y. Chi, M-W. Chung, C-C. Lin. Harvesting luminescence via harnessing the photophysical properties of transition metal complexes. Coord Chem Rev 2011; 255:2653-2665.

[13] H. Xu, R. Chen, Q. Sun, W. Lai, Q. Su, W. Huang, X. Liu. Recent progress in metalorganic complexes for optoelectronic applications. Chem Soc Rev 2014;43:3259-3302.

[14] J. Zhang, D. Ding, Y. Wei, H. Xu. Extremely condensing triplet states of DPEPO-type hosts through constitutional isomerization for high-efficiency deep-blue thermally activated delayed fluorescence diodes. Chem Sci 2016;7:2870-2882.

[15] P.-I. Shih, C.-Y. Chuang, C.-H. Chien, E. W.-G. Diau, C.-F. Shu. Highly Efficient Non-Doped Blue-Light-Emitting Diodes Based on an Anthrancene Derivative End-Capped with Tetraphenylethylene Groups. Adv Funct Mater 2007;17:3141-3146.

[16] Y. H. Kim, D. C. Shin, S.-H. Kim, C.-H. Ko, H.-S. Yu, Y.-S. Chae, S. K. Kwon. Novel blue emitting material with high color purity. Adv Mater 2001;13:1690-1693.

[17] C.-H. Wu, C.-H. Chien, F.-M. Hsu, P.-I. Shih, C.-F. Shu. Efficient non-doped blue-lightemitting diodes incorporating an anthracene derivative end-capped with fluorene groups. J Mater Chem 2009;19:1464-1470. 
[18] F. Liu, W.-Y. Lai, C. Tang, H.-B. Wu, Q.-Q. Chen, B. Peng, W. Wei, W. Huang, Y. Cao. Synthesis and Characterization of Pyrene-Centered Starburst Oligofluorenes. Macromol Rapid Commun 2008;29:659-664.

[19] C. Adachi, T. Tsutsui, S. Saito. Blue light-emitting organic electroluminescent devices. Appl Phys Lett 1990;56:799-801.

[20] S. Tao, Y. Zhou, C.-S. Lee, S.-T. Lee, D. Huang, X. Zhang. Highly efficient non-doped blue organic light-emitting diodes based on anthracene-triphenylamine derivatives. J Phys Chem C 2008;112:14603-14606.

[21] S.-K. Kim, B. Yang, Y.-I. Park, Y. Ma, J.-Y. Lee, H.-J. Kim, J. Park. Synthesis and electroluminescent properties of highly efficient anthracene derivatives with bulky side groups. Org Electron 2009;10:822-833.

[22] W. Staroske, M. Pfeiffer, K. Leo, M. Hoffmann. Single-step triplet-triplet annihilation: An intrinsic limit for the high brightness efficiency of phosphorescent organic light emitting diodes. Phys Rev Lett 2007;98:197402.

[23] N. C. Giebink, S.R. Forrest. Quantum efficiency roll-off at high brightness in fluorescent and phosphorescent organic light emitting diodes. Phys Rev B Condens Matter Mater Phys 2008;77:235215.

[24] Y-J. Pu, G. Nakata, F. Satoh, H. Sasabe, D. Yokoyama, J. Kido. Optimizing the Charge Balance of Fluorescent Organic Light-Emitting Devices to Achieve High External Quantum Efficiency Beyond the Conventional Upper Limit. Adv Mater 2012;24:1765-1770. 
[25] J. H. Jou, S. Kumar, M. Singh, Y.H. Chen, C.C. Chen, M.T. Lee. Carrier Modulation LayerEnhanced Organic Light-Emitting Diodes. Molecules 2015;20:13005-13030.

[26] M. Sun, L. Zhu, W. Kan, Y. Wei, D. Ma, X. Fan, W. Huang, H. Xu. Ternary donoracceptor phosphine oxide hosts with peculiar high energy gap for efficient blue electroluminescence. J Mater Chem C 2015;3:9469-9478.

[27] Y. Yuan, J. Chen, F. Lu, Q. Tong, Q. Yang, H. Mo, T-W. Ng, F. Wong, Z. Guo, J. Ye, Z. Chen, X-H. Zhang, C-S. Lee. Bipolar Phenanthroimidazole Derivatives Containing Bulky Polyaromatic Hydrocarbons for Nondoped Blue Electroluminescence Devices with High Efficiency and Low Efficiency Roll-Off. Chem Mater 2013;25:4957-4965.

[28] K. Wang, S. Wang, J. Wei, Y. Miao, Y. Liu, Y. Wang. Novel diarylboranephenanthroimidazole hybrid bipolar host materials for high-performance red, yellow and green electrophosphorescent devices. Org Electron 2014;15:3211-3220.

[29] N. Su, F. Meng, J. Chen, Y. Wang, H. Tan, S. Su, W. Zhu. Near-infrared emitting pyrazolebridged binuclear platinum complexes: Synthesis, photophysical and electroluminescent properties in PLEDs. Dyes \& Pigments 2016;128:68-74.

[30] H. Liu, P. Chen, D. Hu, X. Tang, Y. Pan, H. Zhang, W. Zhang, X. Han, Q. Bai, P. Lu, Y. Ma. Separation of Electrical and Optical Energy Gaps: Selectively Adjusting the Electrical and Optical Properties for a Highly Efficient Blue Emitter. Chem Eur J 2014;20:2149-2153. 
[31] C.H. Chen, J. Shi. Solution-Processible Multi-component Cyclometalated Iridium Phosphors for High-Efficiency Orange-Emitting OLEDs and Their Potential Use as White Light Sources. Chem Rev 1998;171:161-174.

[32] W. Kan, L. Zhu, Y. Wei, D. Ma, M. Sun, Z. Wu, W. Huang, H. Xu. Phosphine oxide-jointed electron transporters for the reduction of interfacial quenching in highly efficient blue PHOLEDs. J Mater Chem C 2015;3:5430-5439.

[33] M. Liu, X-L. Li, D. Chen, Z. Xie, X. Cai, G. Xie, K. Liu, J. K.;Tang, S-J. Su, Y. Cao. Study of Configuration Differentia and Highly Efficient, Deep-Blue, Organic Light-Emitting Diodes Based on Novel Naphtho [1, 2-d] imidazole Derivatives. Adv Funct Mater 2015;25:5190-5198.

[34] Y. Zhang, S-L. Lai, Q-X. Tong, M-F. Lo, T-W. Ng, M-Y. Chan, Z-C. Wen, J. He, K-S. Jeff, X-L. Tang, W-M. Liu, C-C. Ko, P-F. Wang, C-S. Lee. High efficiency nondoped deep-blue organic light emitting devices based on imidazole- $\pi$-triphenylamine derivatives. Chem Mater 2012;24:61-70.

[35] Z. Gao, G. Cheng, F. Shen, S. Zhang, Y. Zhang, P. Lu, Y. Ma. Highly efficient deep blue light emitting devices based on triphenylsilane modified phenanthro [9, 10-d] imidazole. Laser Photonics Rev 2014;8:L6-L10.

[36] W. Qin, Z. Yang, Y. Jiang, J. W.Y. Lam, G. Liang, H.S. Kwok,B.Z. Tang. Construction of efficient deep blue aggregation-induced emission luminogen from triphenylethene for nondoped organic light-emitting diodes. Chem Mater 2015;27:3892-3901. 
[37] X. Ouyang, X. Li, L. Ai, D. Mi, Z. Ge, S-J Su. Novel "Hot Exciton” Blue Fluorophores for High Performance Fluorescent/Phosphorescent Hybrid White Organic Light-Emitting Diodes with Superhigh Phosphorescent Dopant Concentration and Improved Efficiency Roll-Off. ACS Appl Mater Interfaces 2015;7:7869-7877.

[38] D. Kumar, K.R. Thomas, C.C. Lee, K.C. Ho. Novel pyrenoimidazole-based organic dyes for dye-sensitized solar cells. Org Lett 2011;13:2622-2625.

[39] D. Kumar, K. Thomas, C.P. Lee, K.C. Ho. Triarylamine-Free Pyrenoimidazole-Containing Organic Dyes with Different $\pi$-Linkers for Dye-Sensitized Solar Cells. Asian J Org Chem 2015;4:164-172.

[40] P.C. Bevilacqua, R. Kierzek, K.A. Johnson and D.H. Turner. P.C. Bevilacqua, R. Kierzek, K.A. Johnson, D.H. Turner. Dynamics of ribozyme binding of substrate revealed by fluorescence-detected stopped-flow methods. Science 1992;258:1355.

[41] W.L. Jia, T. McCormick, Q.D. Liu, H. Fukutani, M. Motala, R.Y. Wang, Y. Tao, S.N. Wang. Diarylamino functionalized pyrene derivatives for use in blue OLEDs and complex formation. J Mater Chem 2004;14:3344.

[42] W. Li, D. Liu, F. Shen, D. Ma, Z. Wang, T. Feng, Y. Xu, B. Yang, Y. Ma. A Twisting Donor-Acceptor Molecule with an Intercrossed Excited State for Highly Efficient, Deep-Blue Electroluminescence. Adv Funct Mater 2012;22:2797-2803. 
[43] X. Ouyang, X-L. Li, Y. Bai, D. Mi, Z. Ge, S-J. Su. Highly-efficient hybrid white organic light-emitting diodes based on a high radiative exciton ratio deep-blue emitter with improved concentration of phosphorescent dopant. RSC Adv 2015;5:32298-32306.

[44] Y-C. Chang, S-C. Yeh, Y-H. Chen, C-T. Chen, R-H. Lee, R-J. Jeng. New carbazolesubstituted anthracene derivatives based non-doped blue light-emitting devices with high brightness and efficiency. Dyes Pigments 2013;99:577-587.

[45] M.S. Subeesh, K. Shanmugasundaram, C.D. Sunesh, T.P. Nguyen, Y. Choe. Phenanthroimidazole Derivative as an Easily Accessible Emitter for Non-Doped Light-Emitting Electrochemical Cells. J Phys Chem C 2015;119:23676-23684.

[46] Z. M. Wang, P. Lu, S. M. Chen, Z. Gao, F. Z. Shen, W. S. Zhang, Y. X. Xu, H. S. Kwok , Y. G. Ma. Phenanthro[9,10-d]imidazole as a new building block for blue light emitting materials. J Mater Chem 2011;21:5451.

[47] W. Li, L. Yao, Y. Liu, Z. Wang, S. Zhang, R. Xiao, H. Zhang, P. Lu, B. Yang, Y. Ma. Highly efficient deep-blue OLED with an extraordinarily narrow FHWM of $35 \mathrm{~nm}$ and a y coordinate $<0.05$ based on a fully twisting donor-acceptor molecule. J Mater Chem C 2014;2:4733.

[48] Y. Zhang, S-L. Lai, Q-S. Tong, M-F. Lo, T-W. Ng, M-Y. Chan, Z-C. Wen, J. He, K-S. Jeff, X-L. Tang, W-M. Liu, C-C. Ko, P-F. Wang, C-S. Lee. High Efficiency Nondoped Deep-Blue Organic Light Emitting Devices Based on Imidazole- $\pi$-triphenylamine Derivatives. Chem Mater 2012;24:61-70.

[49] T. Shan, Y. Liu, X. Tang, Q. Bai, Y. Gao, Z. Gao, J. Li, J. Deng, B. Yang, P. Lu,Y. Ma. Highly Efficient Deep Blue Organic Light-Emitting Diodes Based on Imidazole: Significantly 
Enhanced Performance by Effective Energy Transfer with Negligible Efficiency Roll-off. ACS Appl Mater Interfaces 2016;8 (42):28771-28779. 